\title{
Ground-state depletion for subdiffraction-limited spatial resolution in coherent anti-Stokes Raman scattering microscopy
}

\author{
Carsten Cleff, ${ }^{*}$ Petra Groß ${ }^{\dagger}$ and Carsten Fallnich \\ Institute of Applied Physics, Westfälische Wilhelms-Universität Münster, Corrensstrasse 2, 48149 Münster, Germany
}

Herman L. Offerhaus and Jennifer L. Herek

Optical Sciences Group, MESA + Research Institute for Nanotechnology, University of Twente, P.O. Box 217, Enschede NL-7500 AE, The Netherlands

\author{
Kai Kruse, Willem P. Beeker, Chris J. Lee, and Klaus-Jochen Boller \\ Laser Physics \& Nonlinear Optics Group, MESA + Research Institute for Nanotechnology, University of Twente, P.O. Box 217 , \\ Enschede NL-7500 AE, The Netherlands
}

(Received 16 March 2012; published 16 August 2012)

\begin{abstract}
We theoretically investigate ground-state depletion for subdiffraction-limited spatial resolution in coherent anti-Stokes Raman scattering (CARS) microscopy. We propose a scheme based on ground-state depopulation, which is achieved via a control laser light field incident prior to the CARS excitation light fields. This ground-state depopulation results in a reduced CARS signal generation. With an appropriate choice of spatial beam profiles, the scheme can be used to increase the spatial resolution. Based on the density matrix formalism we calculate the CARS signal generation and find a CARS signal suppression by $75 \%$ due to ground-state depletion with a single control light field and by using two control light fields the CARS signal suppression can be enhanced to 94\%. Additional control light fields will enhance the CARS suppression even further. In case of a single control light field we calculate resulting CARS images using a computer-generated test image including quantum and detector noise and show that the background from the limited CARS suppression can be removed by calculating difference images, yielding subdiffraction-limited resolution where the resolution achievable depends only on the intensity used.
\end{abstract}

DOI: 10.1103/PhysRevA.86.023825

PACS number(s): 42.30.-d, 78.20.Bh, 78.47.jh, 87.64.-t

\section{INTRODUCTION}

Far-field optical microscopy is one of the basic techniques for sample analysis in life science. Besides the classical transmission and reflection microscopy, fluorescence microscopy and nonlinear microscopy techniques, such as secondharmonic generation (SHG) [1] and third-harmonic generation (THG) [2], as well as stimulated Raman scattering (SRS) [3,4] and coherent anti-Stokes Raman scattering (CARS) [5,6], have developed rapidly in the recent past. This has led to new opportunities in terms of better image contrast and chemical selectivity. The nonlinear microscopy techniques intrinsically allow three-dimensional (3D) images to be acquired, with the size of the 3D focal volume defining the spatial resolution limit. Resolution enhancement in the lateral dimensions has been demonstrated using linear (interference) methods by designing the spatial excitation profiles, but these attempts were limited to an improvement by a factor of only two [7-9].

In comparison to nonlinear microscopy techniques, fluorescence microscopy suffers from the disadvantage that it requires staining in order to achieve contrast, which comes with problems such as being cytotoxic, modifying the sample or lacking of specific binding to one component. However, due to the saturable nature of stimulated emission depletion

\footnotetext{
*c.cleff@uni-muenster.de

${ }^{\dagger}$ Present address: Institute for Physics, Carl von Ossietzky Universität, 26111 Oldenburg, Germany.
}

(STED) in fluorescence microscopy, this method enables acquisition of images with spatial resolution significantly beyond the diffraction limit [10]. STED paved the way to a better understanding of intracellular physiological processes; for example, it enabled visualization of the synaptic vesicle movement inside the axons of neurons [11]. Besides biological applications, microscopy techniques with very high spatial resolution are needed for the investigation of nanostructures. However, in contrast to biological samples these structures often cannot be stained. An autofluorescence in such materials, if present at all, is often based on defects, meaning that structures with low defect rates are hardly visible with fluorescence microscopy and STED is hardly applicable. Therefore, nonlinear microscopy techniques, such as CARS microscopy, are of central interest for these investigations [12]. Although nonlinear microscopy techniques offer a higher spatial resolution compared to linear techniques, due to the nonlinear point spread function, the resolution is still limited by diffraction.

In order to also provide subdiffraction-limited spatial resolution in far-field CARS microscopy, we present a theoretical investigation of a scheme for a saturable suppression of the CARS signal generation. In CARS microscopy two pulsed light fields, the pump field with frequency $\omega_{p}$ and the Stokes field with frequency $\omega_{S t}$, are used to coherently transfer population to the Raman state via a two-photon resonant Raman active vibrational transition with frequency $\Omega_{v i b}=$ $\omega_{p}-\omega_{S t}$. The vibrational coherence between the ground state and the Raman state is probed by a third pulsed light field with frequency $\omega_{p r}$, generating a new frequency-shifted light 
field at $\omega_{C A R S}=\omega_{p}-\omega_{S t}+\omega_{p r}$. In our previous work, we showed that it should be possible to suppress the buildup of a CARS signal by preventing a coherence between the ground state and the Raman state. In [13], suppression of the CARS signal was demonstrated by considering population of a vibrational state with a strong incoherent coupling to the Raman state and in [14] sidebands of the CARS frequency with an intensity-dependend frequency shift were created by a coherently driven ground-state population oscillation. Both schemes require rather specific molecular properties, that is, high incoherent coupling between a vibrational and a Raman state in the first scheme and long coherence lifetimes in the second scheme, which restricts the applicability of the methods. Compared to our previous calculations, the work presented here describes a more general approach.

Here, we only consider effectively incoherent population transfer, which can be achieved by stimulating transitions between two states with an optical field with a pulse duration longer than the lifetime of the coherence between those states. This is generally possible without further restrictions on the molecular properties. In addition, we do not require nonradiative transitions between states, making this approach applicable to a wide range of molecules. Our approach is based on depletion of the ground-state population distribution of the sample. We numerically study the effect of a control light field on depleting the ground-state population density and investigate the influence on CARS signal generation. We show how even partial CARS signal depletion can be used to obtain subdiffraction-limited spatial resolution using a donut-shaped control beam as it is typically used in STED microscopy. In contrast to a spatial resolution enhancement resulting from designing the pump, Stokes and probe beam profiles [8,9], our approach offers a spatial resolution that depends only on the control light field intensity. Thereby, the spatial resolution can easily be matched to the feature scale of individual samples.

\section{THEORETICAL FRAMEWORK}

In order to investigate the CARS signal generation with applied control light fields, we used a density matrix formalism approach to describe a four-level system shown in Fig. 1. The CARS signal is calculated from the off-diagonal elements of the density matrix. A detailed description of the density matrix

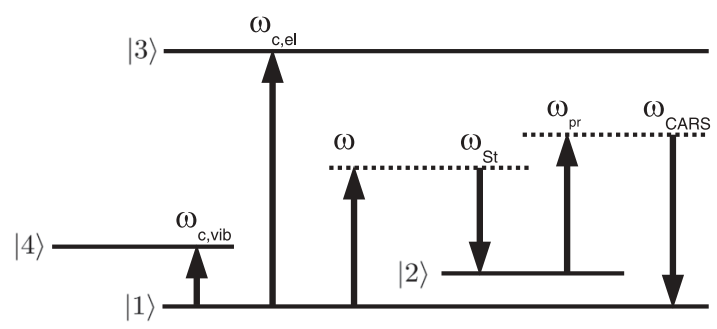

FIG. 1. Energy diagram of the CARS process. The ground state $|1\rangle$ is initially fully occupied. State $|2\rangle$ is a Raman-active vibrational state and $|3\rangle$ is the upper electronic state acting as control state. An additional control state is included by adding an infrared-active vibrational state $|4\rangle$. A control light field resonant with the vibrational state $|4\rangle$ or the electronic level $|3\rangle$ is used to reduce the ground-state population and, thereby, to suppress the CARS signal generation. formalism and its evaluation to calculate the amplitude of the CARS signal is given in [13]. The level system consists of the ground state $|1\rangle$, a vibrational state $|2\rangle$ involved in the CARS process (to which we refer as Raman state), an excited electronic state $|3\rangle$, and a second vibrational state $|4\rangle$. Transitions from the ground state to the vibrational and electronic state as well as the transition from the electronic state to the Raman state are dipole allowed while all other transitions are dipole forbidden. Either one of the electric $|3\rangle$ and vibrational states $|4\rangle$ or both (Sec. III B) are used as control states for ground-state depletion, thereby covering control light field frequencies from the UV to the mid-IR. Infrared-active transitions are usually found in close proximity to the Raman-active transitions in the energy level schemes of molecules, and, therefore, are a general property of complex molecules [15]. The arrows in Fig. 1 indicate the applied and generated light fields: The pump $\left(\omega_{p}\right)$ and Stokes $\left(\omega_{S t}\right)$ light fields are chosen to be two-photon resonant with a transition from $|1\rangle$ to $|2\rangle$, driving population from the ground state to the Raman state, and induce a coherence $\rho_{21}$ between both states, which is essential for the generation of a CARS signal. The probe light field $\left(\omega_{p r}\right)$ interacts with the generated coherence between the ground state $|1\rangle$ and the Raman state $|2\rangle$ generating a sideband at the CARS frequency $\omega_{C A R S}$.

In order to deplete the signal generation in the CARS process we have simulated irradiation of a sample with a control light field prior to the light fields for CARS signal generation. The CARS signal depletion is based on groundstate depopulation to a control state by the control light field. The reduced ground-state population leads to a reduced population transfer to the Raman state $|2\rangle$ and a reduced buildup of vibrational coherence $\rho_{21}$ during the CARS process, resulting in a reduced signal generation. To achieve a saturation of the depletion of the CARS signal and avoid back transfer of population to the ground state $|1\rangle$ due to, for example, Rabi oscillations, the pulse duration of the control light field is chosen sufficiently long (with respect to the lifetime of the coherence between the ground and control states), thereby obtaining an effectively incoherent population transfer. In the following we thus speak of incoherent population transfer. We have considered two cases of control states: first, irradiation by the control light field $\omega_{c, v i b}$, which was resonant with the transition $|1\rangle-|4\rangle$, thus leading to a population transfer from the ground state to the vibrational state $|4\rangle$, and second, irradiation by the control light field $\omega_{c, e l}$, analogously leading to a population transfer from the ground state to the electronic state $|3\rangle$.

Note that we did not consider states with higher transition frequencies than to the electronic states, especially vibrational states coupled to the electronic state. Such vibrational states could also be populated during the CARS excitation and lead to a CARS signal when the electronic state shows a significant population, known as excited-state CARS [16]. However, the transition frequency between such a vibrational state and the electronic state $|3\rangle$ is different compared to the transition frequency between the Raman state $|2\rangle$ and the ground state $|1\rangle$ [17]. Therefore, when sufficiently narrow bandwidth pump and Stokes light fields are used, such as Fourier-limited picosecond pulses, no excited-state CARS signal is generated when a CARS signal is generated from the ground-state 
population due to the different transition frequencies. Thus, for simplicity, higher states than the electronic state can be neglected.

For the calculations we choose parameters that are typical for molecules and CARS experiments. The transition from the ground state to an electronic state is typically in the UV, which we represent by choosing the $|1\rangle-|3\rangle$ transition frequency to $1000 \mathrm{THz}(300 \mathrm{~nm})$. The $|1\rangle-|2\rangle$ transition between the ground state and the Raman state is set to $47 \mathrm{THz}\left(1568 \mathrm{~cm}^{-1}\right.$, $6379 \mathrm{~nm}$ ) and the $|1\rangle-|4\rangle$ transition between the ground state and the vibrational state to $97 \mathrm{THz}\left(3236 \mathrm{~cm}^{-1}, 3091 \mathrm{~nm}\right)$ [15]. The population lifetimes of the states $|2\rangle,|3\rangle$, and $|4\rangle$ are chosen to be $1 \mathrm{~ns}$ [18-20], while the coherence lifetimes are 1 ps for the electronic state $|3\rangle$ and 5 ps for the vibrational state $|4\rangle$ as well as the Raman state $|2\rangle$ [21,22]. Here, for generality, no nonradiative transition between states $|2\rangle$ and $|4\rangle$ is assumed, but the effect of such a transition is investigated separately (Sec. III C). The parameters used for the energy level scheme as well as for the life and coherence times are common, such that the assumed properties should be easy to realize. Thus, the results obtained in the following can be considered to be general and applicable to a broad range of molecules, thereby yielding an improvement over our previous rather specific calculations $[13,14]$.

The pump, Stokes, and probe light field frequencies are set to $335 \mathrm{THz}(895 \mathrm{~nm}), 288 \mathrm{THz}(1040 \mathrm{~nm})$, and $395 \mathrm{THz}$ $(759 \mathrm{~nm})$, respectively. The pulse durations of the three light fields are $2 \mathrm{ps}$ (half width at $1 / e^{2}$ amplitude). The control light field frequency is set to either $\omega_{c, v i b}=97 \mathrm{THz}$ or $\omega_{c, e l}=$ $1000 \mathrm{THz}$ to be resonant with a transition from the ground state to the vibrational or electronic state, respectively. The duration of the control pulse is $10 \mathrm{ps}$ and its pulse peak intensity arrives at the sample $30 \mathrm{ps}$ prior to the peaks of the pump, Stokes, and probe light fields. The numerical calculations are performed using a Runge-Kutta algorithm of fourth order with fixed step size of $0.5 \mathrm{fs}$ and extend over a time period of $100 \mathrm{ps}$.

As the oscillator strengths of the transitions vary strongly for different molecules, in our calculations they are set to a fixed but somewhat arbitrary value. It turns out that only the achieved population transfer is important for the results. Thus, the intensity values of the light fields are given in arbitrary units and in Sec. III F the intensity values needed for an experimental realization are estimated for different samples.

\section{RESULTS}

\section{A. Ground-state depletion}

The diagonal elements $\rho_{11}, \rho_{22}, \rho_{33}$, and $\rho_{44}$ of the density matrix at the end of each calculation (extending over $100 \mathrm{ps}$ ), which are proportional to the final population density of the states, are shown in Fig. 2(a) as a function of the control light intensity for resonance of the control light field with the electronic level $|3\rangle$ and in Fig. 2(b) for resonance with the vibrational level $|4\rangle$. In both cases the targeted state's population can be seen to increase strongly with the control light field intensity to an intermediate population density of approximately 0.5 as it is expected for incoherent population transfer in a two-level system. While the population transfer would completely saturate at an equal population of the control and ground state in a two-level system, in the four-level system the saturation at 0.5 is only intermediate. Thereby, different ranges of control intensities at which an intermediate saturation occurred were observed for the vibrational and electronic state. For the electronic state $|3\rangle$ the intermediate saturation spanned over 2 orders of magnitude of the control intensity [Fig. 2(a)] and over 6 orders of magnitude for a resonance with the vibrational state $|4\rangle$ [Fig. 2(b)]. A further increase of the control intensity stimulates off-resonance population transfer between the states $|3\rangle-|2\rangle$ and $|4\rangle-|2\rangle$, respectively, leading to an additional population of the Raman state $|2\rangle$ and eventually to an equal distribution of the population between these three states. With even higher intensities, it was found that population also enters the fourth state [which is $|4\rangle$ in Fig. 2(a) and $|3\rangle$ in Fig. 2(b)], resulting in the population being distributed equally between all four states.

The CARS signal as a function of the control light field intensity is shown in Fig. 2(c) for the two cases, that the control light field is in resonance with the vibrational state |3〉 (red curve) or that it is in resonance with the electronic state $|4\rangle$ (black curve). It can be seen that when the ground state is depleted by $50 \%$, the CARS signal drops to $25 \%$ of the undepleted CARS intensity $I_{C A R S, 0}$. This can be understood as a result of the coherent nature of the CARS process, for which the CARS intensity is proportional to the square of the population in the focus volume. It can be seen that the CARS intensity remains at $25 \%$ for a certain range of control light intensity, which is larger for the case of the vibrational
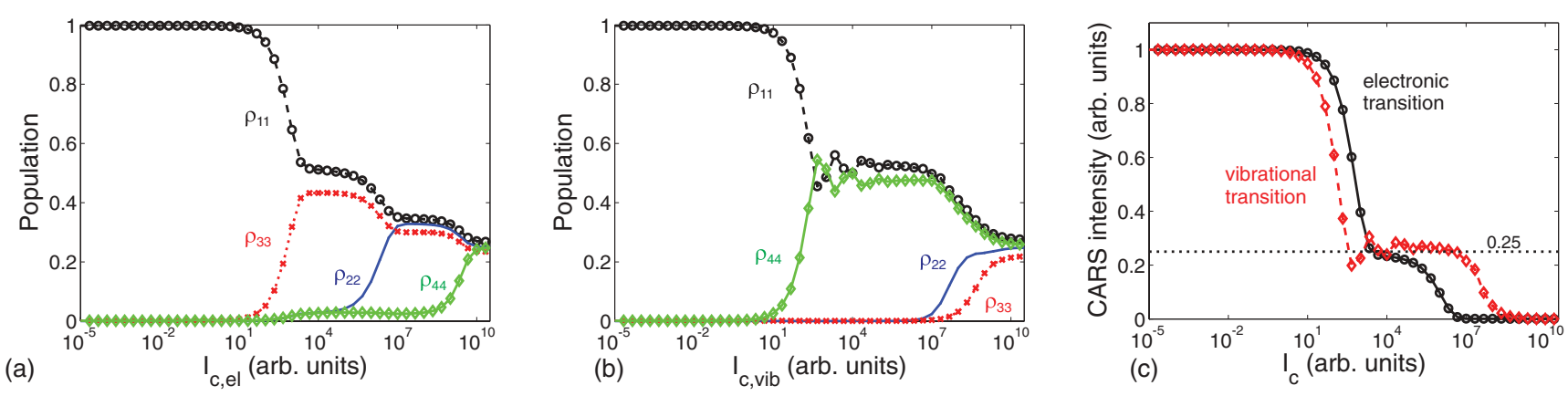

FIG. 2. (Color online) (a) Population of the states $|1\rangle$ (black, $\rho_{11}$ ), $|2\rangle$ (blue, $\rho_{22}$ ), $|3\rangle$ (red, $\rho_{33}$ ), and $|4\rangle$ (green, $\rho_{44}$ ) as a function of the control laser intensity $I_{c, e l}$ in resonance with the $|1\rangle-|3\rangle$ transition. (b) The population of the levels as a function of $I_{c, v i b}$ in resonance with $|1\rangle-|4\rangle$ transition. (c) CARS intensity as a function of the control laser intensity $I_{c}$ resonant with the $|1\rangle-|3\rangle$ (black solid curve) or $|1\rangle-|4\rangle$ (red dashed curve) transition. 
resonance of the control field as compared to the case of electronic resonance. This is in agreement with the respective intensity ranges within which the ground state is depleted by $50 \%$ [see Figs. 2(a) and 2(b)]. At higher control light field intensities, when the population of the Raman state $|2\rangle$ becomes significant, the CARS signal is, consequently, more strongly suppressed. In both cases, the additional suppression is due to a further reduction in population difference $\rho_{11}-\rho_{22}$ between the ground state and the Raman state as a significant amount of population enters the Raman state $|2\rangle$. Finally, when the populations of the ground and the Raman states were equalized, a CARS signal suppression by more than $99.95 \%$ is reached.

Employing such suppression would offer subdiffractionlimited CARS images without the need of schemes for a further background subtraction as the CARS signal could be suppressed almost completely. However, the occurrence of off-resonance population transfer strongly depends on the detuning of the light field frequency used from the transition frequency as well as on the oscillator strength of the transitions. The different light field frequencies result in a different onset of off-resonance population transfer for the two different control states: When the electronic state is used as control state, an off-resonance population transfer to the Raman state |2) starts almost simultaneously with the resonant population transfer from the ground state $|1\rangle$ to the electronic state $|3\rangle$ [Fig. 2(a)]. In contrast, for the case of the vibrational state acting as control state, an off-resonance population transfer to the Raman state $|2\rangle$ occurs with a control light field intensity higher by some orders of magnitude [Fig. 2(b)]. An additional factor to the onset of off-resonance population transfer is the oscillator strength of the transitions. In our calculation these are taken to be the same for all transitions as they vary strongly for different molecules. However, in general, a transition between the ground state and the control state is stronger than the transition from the control state to a lower vibrational state, which is not taken into account here. This results in even higher control light field intensities to achieve off-resonance population transfer. Therefore, it cannot generally be expected that off-resonance transitions are found, but a saturation of the population of the control state around 0.5 and thus a CARS signal suppression of $75 \%$. As a result of this consideration we do not take into account off-resonance population transfer when we calculate spatial CARS excitation profiles in Sec. IIIE but assume a saturation of the CARS signal suppression at $75 \%$ (in case of a single control light field), rendering a worst-case scenario. While this seems to limit the application for subdiffraction-limited resolution we show in Sec. III E that for a resolution enhancement only a saturation of the CARS signal depletion is necessary and that a higher depletion only improves the tolerable noise levels.

\section{B. Two-color ground-state depletion}

So far we have shown that if the ground state is coupled to a single higher state with one control light field, it can only be depleted by $50 \%$ in case that no additional nonresonant transitions occur, which results in a CARS signal depletion down to $25 \%$. Here we show that, by using a number of control light pulses with different frequencies to excite the

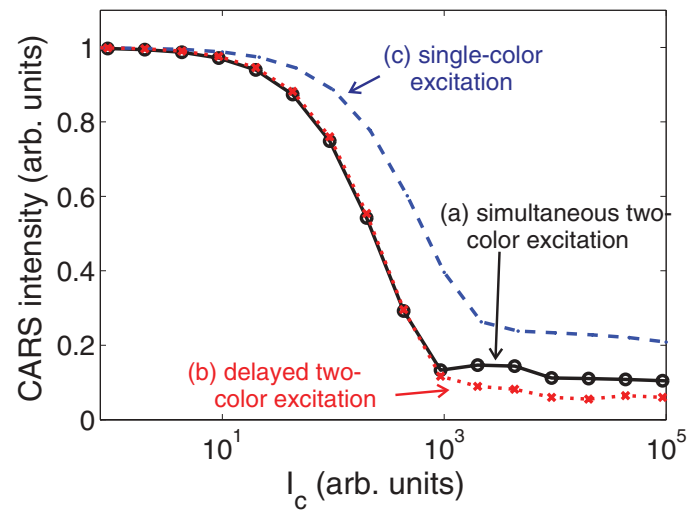

FIG. 3. (Color online) CARS intensity as a function of the control intensity for three cases. (a) Two simultaneously irradiated control light fields, one resonant with the transition to the electronic state $(|1\rangle-|3\rangle)$ and one resonant with the transition to the vibrational state $(|1\rangle-|4\rangle)$ leading to a suppression of the CARS signal by $90 \%$ (black solid curve). (b) A subsequent irradiation of two control light fields, the first one resonant with the $|1\rangle-|3\rangle$ transition and the second one resonant with the $|1\rangle-|4\rangle$ transition, irradiated 20 ps delayed to each other resulted in a ground-state depletion of $75 \%$ and a CARS signal suppression of $94 \%$ (red dotted curve). (c) For comparison a single control light field resonant with the $|1\rangle-|3\rangle$ transition, suppressing the CARS signal by $75 \%$ (blue dashed curve).

ground-state population to $N$ higher states, the CARS signal suppression can be increased significantly. Specifically, in this section we investigate the CARS signal suppression for the case of $N=2$; that is, two control light fields are used, one resonant with the transition $|1\rangle-|3\rangle$ to the electronic state $|3\rangle$ and one resonant with the transition $|1\rangle-|4\rangle$ to the vibrational state $|4\rangle$. Note that one can equally well consider the excitation of two vibrational states (instead of one vibrational and one electronic state). In this case of multiple control light field pulses we expect that it is of great importance whether the control light fields are irradiated simultaneously or delayed to each other, which is motivated as follows: We expect that a simultaneous irradiation results in an equal population of the vibrational and electronic state, depleting the ground state to $33 \%\left(\frac{1}{N+1}\right.$ with $\left.N=2\right)$, leaving a CARS signal of $11 \%$. In contrast, if the two control light fields are delayed to each other, we expect, that the ground state is first depleted by $50 \%$ to one of the higher states by the pulse that arrives first and can thereafter be depleted by $50 \%$ again to the other higher state by the second control pulse leaving a ground-state population of $25 \%\left(\frac{1}{2^{N}}, N=2\right)$ and a CARS signal of $6.25 \%$.

Figure 3 shows the CARS signal as a function of the control light field intensity for three cases. The first two are (a) a simultaneous irradiation with two control light fields (black curve) and (b) irradiation with two control light fields when the transition to the vibrational state $|4\rangle$ is stimulated $20 \mathrm{ps}$ after the transition to the electronic state $|3\rangle$ (red curve). For comparison the case of a single control light field resonant with the electronic state is also shown in Fig. 3 (blue curve). It can be seen that while with a single-color scheme the CARS signal saturates at $0.25 \times I_{C A R S, 0}$, it saturates at $0.10 \times I_{C A R S, 0}$ with the simultaneous irradiation of two control light fields (black curve). A suppression to less than $0.06 \times I_{C A R S, 0}$ is 

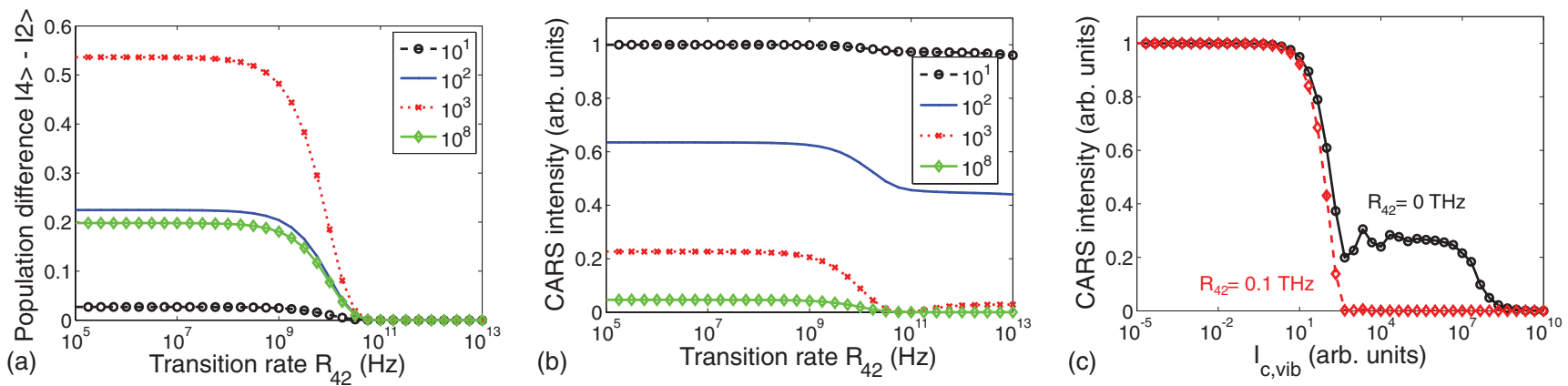

FIG. 4. (Color online) (a) Population difference between the vibrational state $|4\rangle$ and the Raman state $|2\rangle$ for different intensities of the control beam as a function of the nonradiative transition rate $R_{42}$ between the vibrational state $|4\rangle$ and the Raman state $|2\rangle$; (b) resulting depletion of the CARS signal. (c) Normalized CARS intensity as a function of the control light field intensity for a nonradiative transition rate $R_{42}$ of $0 \mathrm{THz}$ (black solid curve) and $0.1 \mathrm{THz}$ (red dashed curve).

found with subsequent control pulses (red curve) matching the CARS signal suppression, that is expected from the theoretical considerations made above.

In conclusion, using a multicolor ground-state depletion the suppression of the CARS signal can be enhanced significantly, leading to an improved contrast due to the strongly reduced background signal generation in subdiffraction-limited CARS images due to the limited saturation. However, although CARS suppression is stronger with a multicolor ground-state depletion, for simplicity we restrict the following theoretical investigations to a single control light field (unless explicitly stated otherwise), but all results are also valid qualitatively for the case of multiple control light fields.

\section{Limit cases}

In order to describe a general case without strong restrictions regarding the applicable molecules, we assumed only effectively incoherent population transfer and neglected nonradiative transitions between the vibrational state $|4\rangle$ and the Raman state $|2\rangle$. However, this assumption cannot be matched in all cases; therefore, we discuss briefly two possible exceptions to the scheme in this section, where our general assumption of the molecular properties are violated.

The first case is that the induced population transfer is coherent instead of incoherent, which does not result in a saturation of the population densities, but in an oscillatory behavior. These oscillations are known as Rabi oscillations. In this case, illumination with a strong control light field does not lead to a suppression of the CARS signal but to a generation of sidebands of the CARS signal with an intensity dependent frequency shift. The frequency shift depends directly on the control light field intensity, such that the effect can be used to resolve emitter positions with subdiffraction-limited accuracy [14].

The second case is that of a population transfer without coherent effects from $|1\rangle$ to $|4\rangle$ is achieved, but with a strong incoherent coupling between the vibrational state $|4\rangle$ and the Raman state $|2\rangle$ due to a nonradiative transition [13]. In this case, the Raman state $|2\rangle$ is prepopulated via the vibrational state $|4\rangle$, but without coherence buildup between the states $|1\rangle$ and $|2\rangle$. As this coherence is essential for CARS signal generation, depletion of the CARS signal is even stronger than in the case without an incoherent nonradiative coupling, which we considered in this work. The nonradiative transition rate $R_{42}$ between the vibrational state $|4\rangle$ and the Raman state $|2\rangle$ may vary strongly for different molecules. In order to estimate the implication of this effect, we investigate the population difference $\rho_{44}-\rho_{22}$ between the vibrational state $|4\rangle$ and the Raman state $|2\rangle$ as well as the generated CARS intensity in dependence of the transition rate $R_{42}$ between $|4\rangle$ and $|2\rangle$ for a number of fixed control light field intensities $\omega_{c, v i b}$ resonant with the $|1\rangle$ to $|4\rangle$ transition. The result is shown in Figs. 4(a) and 4(b), where the population difference $\rho_{44}-\rho_{22}$ and the CARS intensity, respectively, are plotted as a function of the nonradiative transition rate $R_{42}$. The chosen intensities of $10^{1}, 10^{2}, 10^{3}$, and $10^{8}$ correspond to the cases that the ground state is depleted by $7.7 \%, 39 \%, 52 \%$, and $69 \%$ [see Fig. 2(b)]. It can be seen that at transition rates below $9 \mathrm{GHz}$ no population transfer between the vibrational states is observed and thus also the CARS intensity remains unchanged. Above $9 \mathrm{GHz}$ the transition rate becomes high enough for a noticeable population transfer to state $|2\rangle$, which leads to an additional suppression of the CARS signal generation for control light fields that introduce a ground-state depletion of about $50 \%$ or less (black, blue, and red curves). At very high control intensities, where the ground state is depleted by much more than $50 \%$ due to off-resonance population transfer (green curve), the vibrational state $|2\rangle$ is already prepopulated at low transition rates. Therefore, the population difference $\rho_{44}-\rho_{22}$ decreases again when off-resonance population transfer occurs, and the effect of a nonradiative transition decreases, too.

Figure 4(c) displays the CARS intensity as a function of the control light field intensity for the case of no nonradiative transition between $|4\rangle$ and $|2\rangle$ (black curve) and for a high nonradiative transition rate $R_{42}$ of $0.1 \mathrm{THz}$ (red curve). Without a nonradiative transition the CARS intensity shows an intermediate saturation at $25 \%$ and for very high control light field intensities off-resonance transitions to the Raman state result in a further suppression of more than $99 \%$. In contrast, in case of a high nonradiative transition rate $R_{42}$, states $|4\rangle$ and $|2\rangle$ are always equally populated, which leads to a CARS suppression of more than $99.95 \%$ at much lower control light field intensities without the occurrence of an intermediate saturation and off-resonance transitions. However, note 

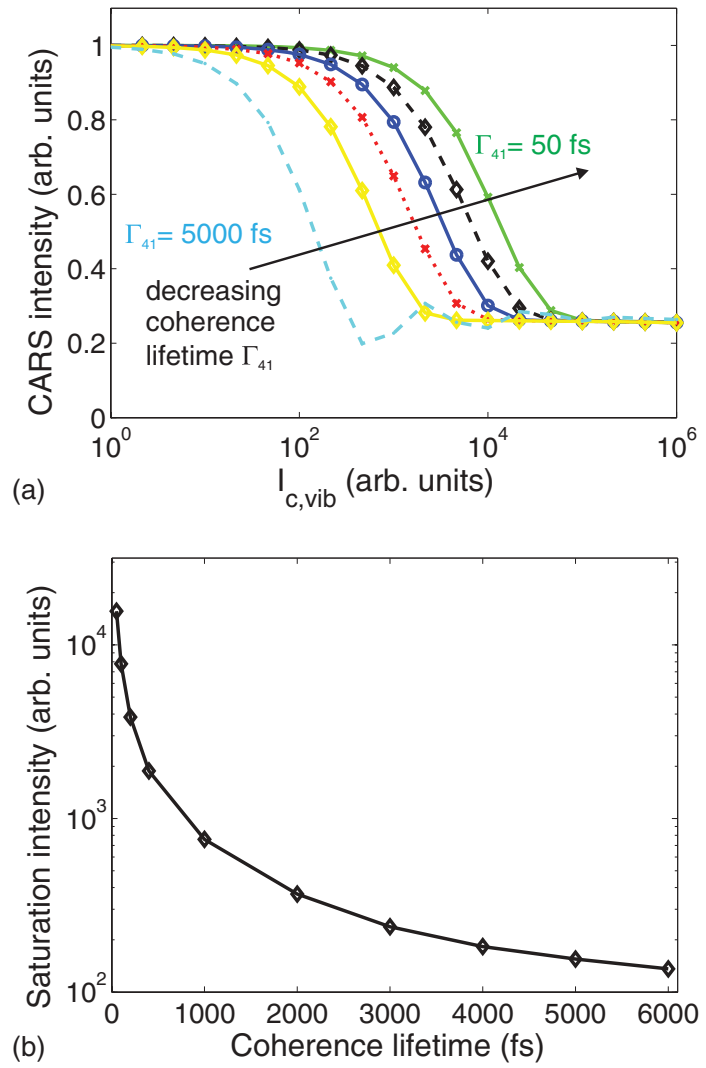

FIG. 5. (Color online) (a) Normalized CARS intensity as a function of the control light field intensity for coherence lifetimes $\Gamma_{41}$ of the vibrational state $|4\rangle$ of 50,100, 200, 400, 1000, and $5000 \mathrm{fs}$. (b) The saturation intensity of the CARS signal suppression decreases with increasing coherence lifetime $\Gamma_{41}$.

that, while high nonradiative transitions between the Raman state and the control state are beneficial for CARS signal suppression, this cannot be expected for arbitrary molecules and control states. To rely on molecular properties that are common for a wide range of molecules we therefore assume only radiative transitions between states and demonstrate that this is sufficient to achieve subdiffraction-limited resolution in CARS microscopy.

\section{Influence of the coherence lifetime}

In Fig. 2(c) we show that there is qualitatively no difference between a ground-state depletion via transitions to a vibrational or electronic state. However, the saturation intensity, where the CARS intensity drops to $50 \%$, differs significantly (by a factor of 5). As the main difference between the two states was the coherence lifetime, in this section we investigate the CARS signal suppression for different coherence lifetimes $\Gamma_{41}$ of the vibrational state for the case that the control light field was resonant with the transition from the ground state to the vibrational state $(|1\rangle-|4\rangle)$.

Figure 5(a) shows the CARS signal as function of the control light field intensity for a number of coherence lifetimes $\Gamma_{41}$ between $50 \mathrm{fs}$ and 5 ps. When $\Gamma_{41}$ is increased, CARS signal suppression appears at lower control light intensities. The saturation intensity as function of $\Gamma_{41}$ is plotted in Fig. 5(b)

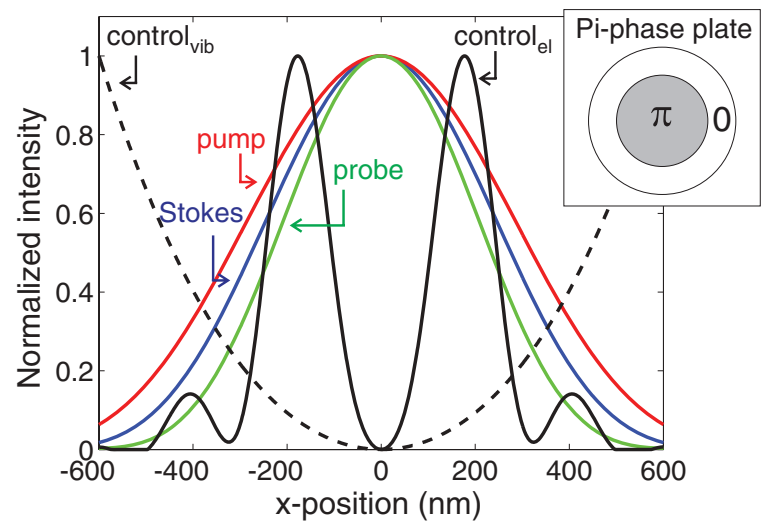

FIG. 6. (Color online) Cross sections of the spatial beam profiles of the pump (red), Stokes (blue), probe (green), and the two control (black) light fields. The inset shows the phase plate used to create the donut-shaped beam profile of the control light fields.

showing the strong monotonous decrease of the saturation intensity with increasing $\Gamma_{41}$.

While a lower saturation intensity is beneficial for subdiffraction-limited CARS microscopy, the accompanying longer coherence lifetime benefits coherent processes which might hamper the scheme. For example, at a coherence lifetime of 5 ps [Fig. 5(a) blue solid curve] we observed that the suppression shows decaying oscillations due to an oscillating population distribution, that is, Rabi-oscillations occurred. This will result in modulations on the point-spread function in CARS microscopy and probably make a background subtraction as explained in Sec. III E more difficult. Choosing a longer pulse duration of, for example, $50 \mathrm{ps}$ for the control light field avoids the occurrence of Rabi-oscillations but the pulse energy required to reach the saturation threshold increases with higher pulse duration. Therefore, to reach the lowest saturation pulse energy the control pulse duration has to be matched to the sample in a way that the pulse is just sufficiently long to avoid oscillations of the population distribution.

\section{E. Beam shape and spatial resolution}

In order to estimate the possible resolution enhancement with the proposed illumination scheme, the spatial beam profiles and the spatial CARS excitation profiles were calculated in two dimensions in the focus plane perpendicular to the direction of beam propagation. To achieve a CARS excitation profile narrower than the diffraction limit an approach well known from STED microscopy was chosen: The control light field is irradiated with a donut-shaped spatial profile while the other light fields are irradiated with a Gaussian spatial profile. Cross sections of the beam profiles in the focal plane are shown in Fig. 6. In this way the pump, Stokes and probe light fields create a CARS excitation profile of Gaussian spatial shape and the control light field suppresses the CARS signal generation in the exterior of the illuminated area without changing the CARS intensity in the center, leaving an excitation profile narrower than the diffraction limit. For the calculations it was assumed that the pump, Stokes, probe, and control light fields are focused through a microscope objective with $\mathrm{NA}=1.0$. The light fields have a Gaussian beam profile before the 


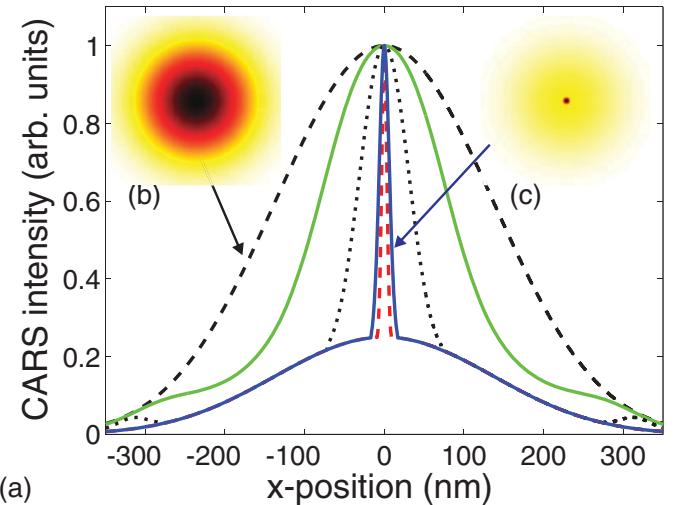

(a)

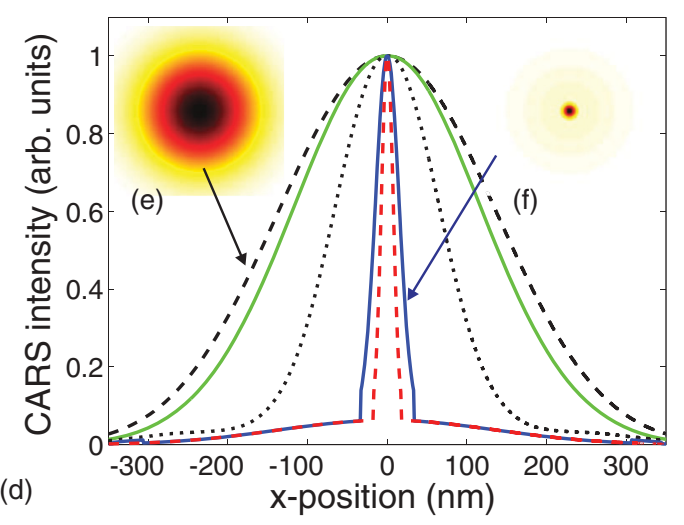

FIG. 7. (Color online) (a) By irradiating a control light field with a donut-shaped spatial profile the CARS excitation profile could be narrowed significantly below the diffraction limit. The achieved width depended directly on the used control light field intensity. The limited suppression of $75 \%$ leaves a significant diffraction-limited background. (d) Using two subsequent control light fields the CARS excitation profiles were similarly narrowed but the background was significantly lower. Two-dimensional illustrations are shown in (b) and (e) for an excitation without control light field and in (c) and (f) for the narrowest excitation profile of each case [(a) and (d) red curve].

microscope objective and the control light field passes through a phase plate before entering the microscope objective. The phase plate adds a phase of $\pi$ to a circular section centered in the beam profile, similar to the one used in [23] (see Fig. 6 inset), thereby generating a donut-shaped control light field in the focal plane. We performed the calculations for two cases: First, a single control light field resonant with the $|1\rangle-|3\rangle$ transition was used, and second, two control light fields were used, the first resonant with the $|1\rangle-|3\rangle$ transition and the second resonant with the $|1\rangle-|4\rangle$ transition, irradiated 20 ps after the first control light field.

As discussed in Sec. III A we assume that no off-resonance population transfer and thus no complete CARS signal suppression can be expected; thus, the CARS signal suppression is limited to $75 \%$ with a single control light field. The excitation profiles using a single donut-shaped control light field with peak intensities of $10^{3}, 10^{4}, 10^{5.4}$, and $10^{6}$ [compare intensities to Fig. 2(c)] are shown in Fig. 7(a). For increasing intensity of the control light field, the CARS excitation profile narrows monotonously. However, due to the saturation of the CARS depletion at $0.25 \times I_{C A R S, 0}$ [see Fig. 2(a)] the excitation profiles lose their initial Gaussian shape and develop into a spatial profile with a narrow upper part (intensity above $0.25 \times I_{C A R S, 0}$ ) and a broader pedestal (intensity below $\left.0.25 \times I_{C A R S, 0}\right)$. Figures $7(\mathrm{~b})$ and $7(\mathrm{c})$ show $2 \mathrm{D}$ illustrations of the initial CARS excitation [Fig. 7(a), black dashed curve] and of a strongly narrowed excitation profile [Fig. 7(a), blue solid curve].

As discussed in Sec. III B, a multicolor ground-state depletion can reduce the CARS signal by much more than 75\%. As an example, we calculated the CARS excitation profile for a multicolor scheme for a number of different peak intensities of the donut-shaped control light fields. The CARS excitation profiles are shown in Fig. 7(d). With increasing control light field intensity the excitation profiles narrow significantly below the diffraction limit but in contrast to Fig. 7(a) a low-resolution background was generated with only $6 \%$ of the original diffraction-limited CARS intensity. For a better visualization 2D illustrations of the diffraction-limited excitation profile [Fig. 7(d) black dashed curve] and the narrowest subdiffraction-limited excitation profile [Fig. 7(d) blue solid curve] are shown in Figs. 7(e) and 7(f), respectively.

In both cases the CARS excitation profile shows a broad pedestal which will create a low-resolution diffraction-limited background in CARS images. Using two control light fields the amplitude of the pedestal is reduced strongly compared to the case of a single control light field and can even be further reduced by additional control light fields. From STED microscopy it is known that, with a pedestal of around $10 \%$, good contrast subdiffraction-limited images can be acquired [24]. Therefore, in the case presented here which involves two subsequent control light fields, where the pedestal is around $6 \%$, no further image processing is necessary for subdiffraction-limited CARS images. In case of a single control light field, the background generated by the pedestal will be too high and additional image processing will be necessary, which will be explained in detail in the following.

Independent of the number of control light fields, the CARS suppression is limited and saturates at a certain level, resulting in the width of the pedestal of the excitation profile to be the same as the diffraction-limited CARS excitation. A quantitative analysis of the width of the excitation profile with one control light field [Fig. 7(a)] is given in Fig. 8. It shows the full width at half maximum (FWHM) of the whole excitation profile and the FWHM of the pedestal (full width at $\left.0.125 \times I_{C A R S, 0}\right)$ as a function of the donut peak intensity. Thereby the width of the full excitation profile corresponds to the lateral resolution that can be achieved. While the FWHM of the full excitation profile monotonously narrows below the diffraction limit, the width of the pedestal reaches a lower limit at the FWHM of the diffraction-limited CARS excitation. Therefore, the background generated by the pedestal of the excitation profile will be shaped like a diffraction-limited CARS image and thus the subdiffraction-limited information can be extracted by calculating the difference image between two CARS images, one taken with and one without an applied control light field.

In order to verify that this scheme is suitable to produce CARS images with a spatial resolution below the diffraction limit we consider a numerically generated test sample [Fig. 9(a)] consisting of a dendritic line structure similar to 


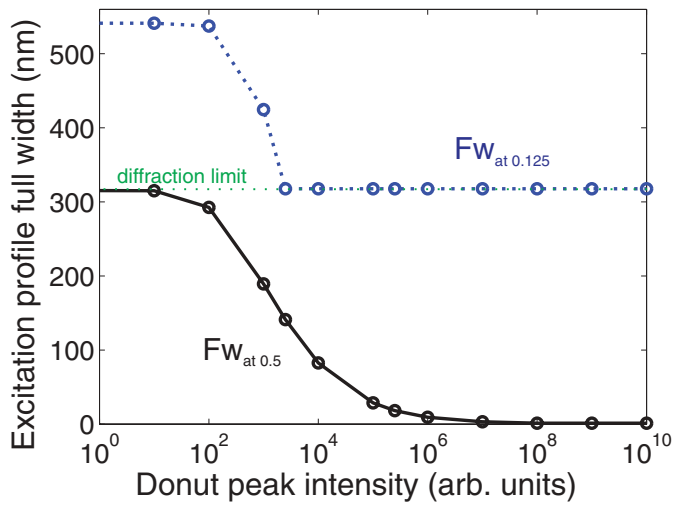

FIG. 8. (Color online) FWHM (black solid line) of the complete excitation profile (corresponding to the achievable lateral resolution) and FWHM of the pedestal (blue dashed line) of the CARS excitation profiles with a single control light field [Fig. 7(a)] as function of the donut peak intensity of the control light field. The width of the pedestal saturates at the diffraction-limit of a CARS excitation which will create a diffraction-limited background below a subdiffractionlimited CARS image.

structures found in, for example, brain tissue. The sample size is $1.34 \times 1.34 \mu \mathrm{m}$, the line thickness $5.4 \mathrm{~nm}$ and the spatial distance between lines less than $540 \mathrm{~nm}$. Emitters are assumed to be homogeneously distributed along the lines. Spatial variations which might result in spatially varying phase terms have not been incorporated. In case such spatially varying phase terms occur, the amplitudes of the CARS light fields from different spatial positions would no longer add up constructively, resulting in a CARS image determined by the spatial variation of the CARS emitters as well as the spatial structure of the phase terms.

However, this would not hamper the presented scheme as the influence of phase terms outside the domain of the subdiffraction-limited peak [Fig. 9(b)] is identical in a subdiffraction-limited and diffraction-limited CARS image and therefore disappears while the difference image calculation. Phase variations within the subdiffraction-limited domain result in phase-dependent CARS amplitude as a result of which the CARS image would no longer provide quantitative information, for example, emitter concentration, unless a phase-sensitive detection is used [25]. This is a limitation that does not result from the applied scheme but is also found in diffraction-limited CARS microscopy as a result of phase variations.

The following calculations are performed using only a single control light field. Note, that the results obtained for this case are also valid for a case with multiple control light fields. Without an applied control light field no features of the test image are visible in the CARS image [Fig. 9(b)], while with an applied strong control light field [red dashed curve in Fig. 7(d), FWHM of $18 \mathrm{~nm}$ ] the CARS image [Fig. 9(c)] shows a strong background, similar to Fig. 9(b), superimposed with a weak subdiffraction-limited image of the test structure. Due to the strong background the subdiffraction-limited features were obscured, but the background could be removed by making a reference measurement without a control light field and by considering the following: Due to the coherent nature of
CARS signal generation the number of emitters in the focal volume contributes to the intensity with a square dependence. This leads to a distorted image when simply subtracting the intensities [Fig. 9(c) from Fig. 9(b)], where isolated features become suppressed [see Fig. 9(d), area at the edges of the image where isolated lines are originally found]. For a more accurate restoration, the electric field amplitudes, that is, the square roots of the measured intensities, have to be subtracted, yielding the image shown in Fig. $9(\mathrm{~g})$ with a spatial resolution corresponding to the width of the narrow spike on top of the CARS excitation profile. In these calculations, no attempt was made to manipulate the intensities of the excitation beams to create a match between the diffraction-lmited pedestal and the diffraction-limited CARS image. Instead, a simple scaling factor was applied to the diffraction-limited CARS image during postprocessing.

Note, that the influence of a nonresonant signal has not been discussed as in this calculation it is found to be only $0.1 \%$ of the resonant signal as a result of the use of picosecond pulse durations. This low nonresonant signal has no noticeable influence on the achieved resolution enhancement. In experimental reports often higher nonresonant backgrounds (of several percent) are found, especially when femtosecond pulses are used. It has also been shown, that with pico- or nanosecond pulses or by employing additional background suppression schemes [25] the nonresonant background can be strongly reduced to levels comparable to our case. However, when the presented CARS depletion scheme is applied in the case that a high nonresonant background is present, this nonresonant background is not reduced by the control beam and leads to image degradation. In the process of the difference image calculation the nonresonant signal is reduced by $25 \%$, leading to the generation of a diffraction-limited background in the difference image. The effect is comparable to the effect of detector noise, which is discussed in the following. Therefore, the limits of an acceptable amount of nonresonant background while maintaining subdiffraction-limited contrast are similar to the limits of acceptable detector noise.

To estimate the applicability of the difference-image method under realistic experimental conditions, we calculate the difference images considering quantum and detector noise. For quantum noise the number of photons $N_{p}$ in each pixel have to be considered, which will introduce a quantum noise level of $\sqrt{N_{p}}$ (considering coherent states). Using longer integration times the number of detected photons can be increased to a level where quantum noise is not noticeable at all, but here we want to give an estimation of experimental conditions which still yield image acquisition times that can be tolerated. In typical CARS experiments we find a photon flux of approximately $10^{6}$ photons/s using a $80-\mathrm{MHz}$ laser system, which we use as basis for our estimations. With an optimized laser system at a lower repetition rate of, for example, $1 \mathrm{MHz}$ using the same average power levels, the CARS photon flux is improved to approximately $6.4 \times 10^{9}$ photons/s without introducing multiphoton damage to the sample [26]. Based on this photon flux estimations we consider integration times between $100 \mathrm{~ms}$ and $100 \mu$ s for laser repetition rates between 80 and $1 \mathrm{MHz}$, respectively, giving a maximum number of photons per pixel of $10^{5}$. Acquiring images with $100 \times 100$ pixels this yields image acquisition times between $17 \mathrm{~min}$ 


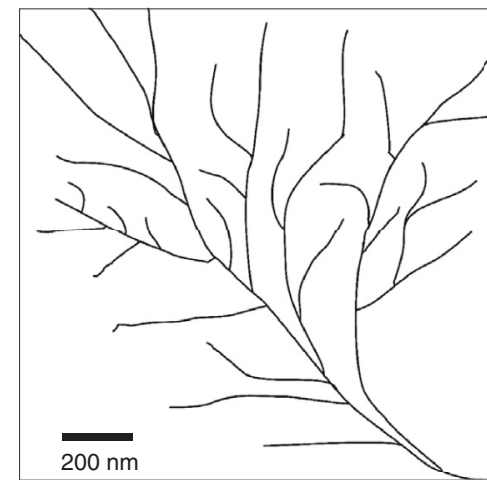

(a)

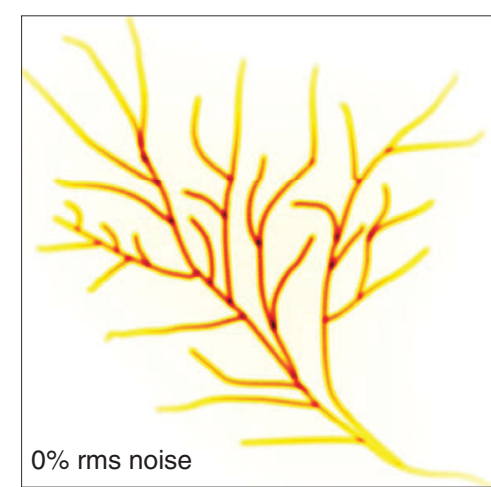

(d)

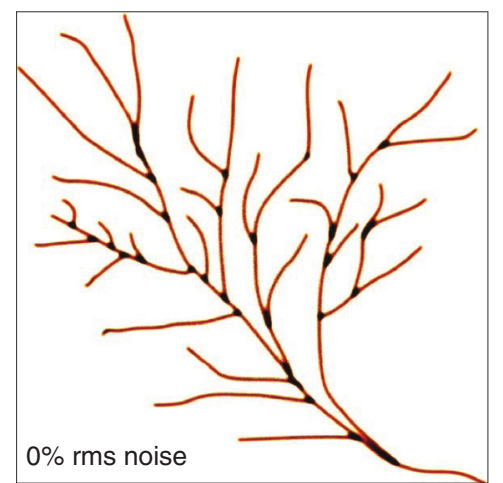

(g)

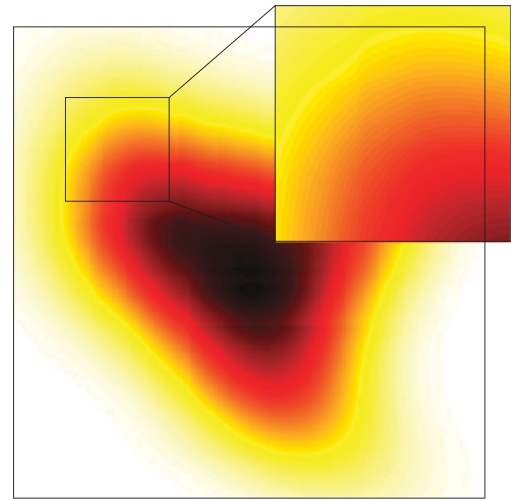

(b)

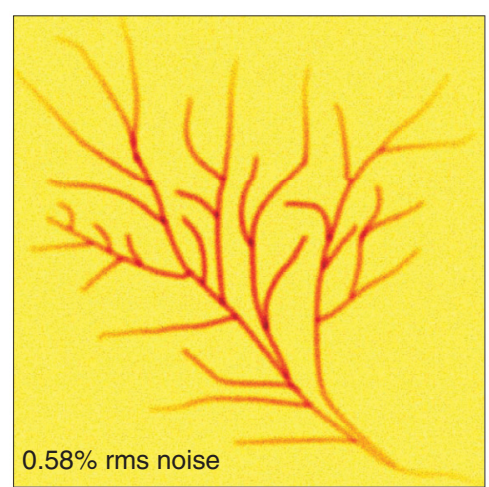

(e)

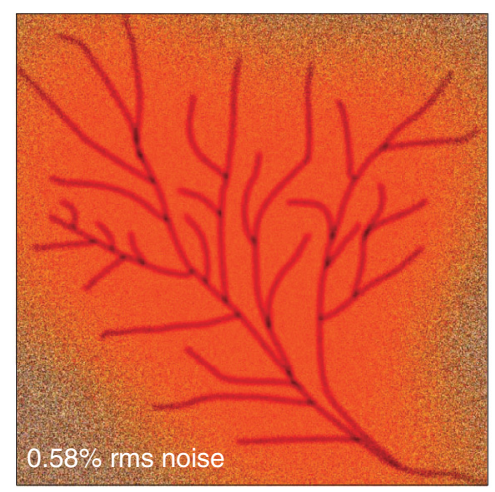

(h)

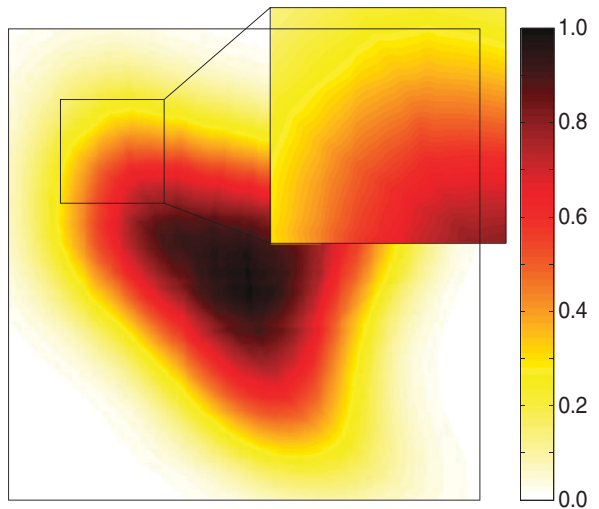

(c)

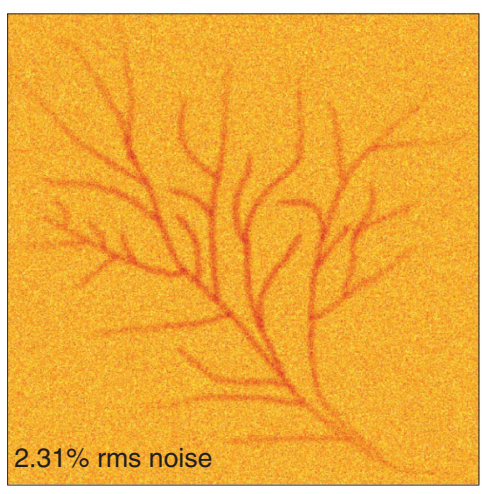

(f)

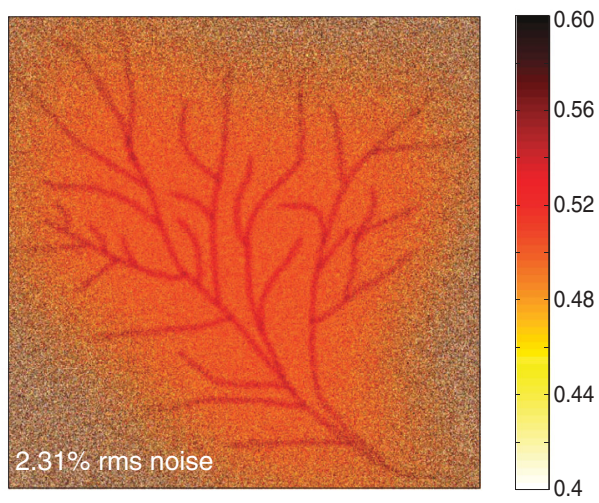

(i)

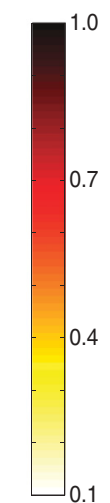

FIG. 9. (Color online) (a) Test sample simulating fine structure of e.g. brain tissue. By calculating the convolution of the test image with the excitation profile, a CARS image was obtained for the case that (b) no control light field was applied and the case (c) that a control light field was applied that resulted in an excitation profile with a FWHM of $18 \mathrm{~nm}$ shown in Fig. 7(c). While in (b) no subdiffraction-limited features are resolved in (c) the fine structure of the test sample is visible on top of the diffraction-limited background. The background can be removed by calculating a difference image between (b) and (c). Due to the coherent nature of the CARS process (d) a difference image from intensity images generates aberrations in the boundary area in contrast to $(\mathrm{g})$ a difference image from the electric field amplitude. As experimental images always contain noise both cases have been considered with (d) and (g) quantum noise only (maximum photon number of $10^{5}$ ) and an additional detector noise of (e) and (h) $0.58 \% \mathrm{rms}$ and (f) and (i) $2.31 \% \mathrm{rms}$.

and $1 \mathrm{~s}$. The considered photon flux features photon number fluctuations of $0.3 \%$, which we find to generate no noticeable reduction of the image contrast [see Figs. 9(d) and 9(g)]. Therefore, the effect of quantum noise will be negligible as soon as detector noise is present.

In order to illustrate the influence of detector noise, which overwhelms the quantum noise in most cases, we consider two noise levels by adding randomly up to 1000 photons $(1.0 \%$ amplitude, $0.58 \% \mathrm{rms}$ ) and 4000 photons
(4\% amplitude, 2.31\% rms) to each pixel in Figs. 9(b) and 9(c), respectively. These values for detector noise are easily achievable in experimental setups even with poor detectors and can be reduced strongly using a detector such as, for example, the photon counting module MP993 (Excelitas Technologies, maximum of $10^{7}$ counts/s with less than 10 dark counts/s). If the difference image is calculated from the CARS intensity images [Figs. 9(b) and 9(c)] the noise adds up linearly allowing a good contrast in the difference image 
[Figs. 9(e), 0.58\% rms noise; and 9(f), $2.31 \%$ rms noise) but with image distortions in the boundary area as discussed above. In case the image reconstruction is calculated from the square root of the intensity images, the image distortions can be avoided, However, the noise is strongly enhanced in the boundary area of the image due to the lower signal-to-noise ratio. Nevertheless, the structure of the test image was still clearly visible [Figs. 9(h), 0.58\% rms noise; and 9(i), $2.31 \%$ rms noise]. Although these noise considerations are rather qualitative they show that the image reconstructions in Fig. 9 are a realistic way to reach an image resolution well below the diffraction limit.

\section{F. Intensity considerations}

The intensity values necessary for a ground-state depletion of $50 \%$ depend strongly on the kind of transition chosen and on the individual transition probabilities. Especially in biological samples these power values are not known but it seems likely that an excitation of a vibrational transition might be the best choice in such a case, because, although the transition probability is lower than for an electronic transition, the damage threshold is typically much higher for biological samples in the IR region. A ground-state depletion by $50 \%$ in a liquid biological sample using mid-IR radiation has been reported by Ventalon et al. [27]. The needed intensity can be estimated to be $200 \mathrm{MW} / \mathrm{cm}^{2}$ for pulses with a duration of $35 \mathrm{ps}$, which is sufficiently low to avoid multiphoton ionization or even multiphoton excitation.

The excitation of an electronic transition might also be practical, especially in the case of nonorganic samples. For example, using CARS microscopy for the investigation of nanostructures [12], such as quantum dots, has become of wide interest. This field has the strong need for higher spatial resolution and the samples under investigation typically show a high damage threshold for frequencies matching the electronic transition. Using, for example, quantum dots even a population oscillation could be demonstrated using a titanium:sapphire laser with peak intensities of less than $70 \mathrm{~kW} / \mathrm{cm}^{2}$ [28].

\section{CONCLUSION}

In conclusion, we have numerically investigated a scheme for subdiffraction-limited spatial resolution in CARS microscopy. The proposed scheme does not need any special molecule properties such as high coherence times or nonradiative (incoherent) coupling between energy states, thus making this approach applicable to a huge variety of molecules. We showed that, upon illuminating the sample with a control light field, a CARS signal can be suppressed by $75 \%$ due to an (effectively) incoherent population transfer from the ground state to a vibrational or electronic state. Using two control light fields to deplete the ground state to two control states promises a suppression of the CARS signal by $94 \%$ which can be enhanced even further by using more control states for ground-state depletion.

We have numerically demonstrated the applicability to subdiffraction-limited CARS microscopy by simulating 2D image acquisition using realistic beam dimensions and a donut-shaped control beam. The resolution was found to be improved far beyond the diffraction limit down to $1.2 \mathrm{~nm}$ and, similar to STED, the practical limit is only the applied power. For the case that the suppression is limited to $75 \%$ we demonstrated that subdiffraction-limited spatial resolution is possible by calculating difference images, which allowed image reconstruction with good contrast while a high noise level was present.

\section{ACKNOWLEDGMENT}

We acknowledge support by the Deutsche Forschungsgemeinschaft and the Open Access Publication Fund of University of Muenster.
[1] R. Hellwarth and P. Christensen, Opt. Commun. 12, 318 (1974).

[2] M. Müller, J. Squier, K. R. Wilson, and G. J. Brakenhoff, J. Microsc. 191, 266 (1998).

[3] E. Ploetz, S. Laimgruber, S. Berner, W. Zinth, and P. Gilch, Appl. Phys. B 87, 389 (2007).

[4] C. W. Freudiger, W. Min, B. G. Saar, S. Lu, G. R. Holtom, C. He, J. C. Tsai, J. X. Kang, and X. S. Xie, Science 322, 1857 (2008).

[5] A. Zumbusch, G. R. Holtom, and X. S. Xie, Phys. Rev. Lett. 82, 4142 (1999).

[6] J.-X. Cheng and X. S. Xie, J. Phys. Chem. B 108, 827 (2004).

[7] O. Masihzadeh, P. Schlup, and R. A. Bartels, Opt. Lett. 34, 1240 (2009).

[8] V. Raghunathan and E. O. Potma, J. Opt. Soc. Am. A 27, 2365 (2010).

[9] H. Kim, G. W. Bryant, and S. J. Stranick, Opt. Express 20, 6042 (2012).

[10] S. W. Hell and J. Wichmann, Opt. Lett. 19, 780 (1994).
[11] V. Westphal, S. O. Rizzoli, M. A. Lauterbach, D. Kamin, R. Jahn, and S. W. Hell, Science 320, 246 (2008).

[12] Y. Wang, C.-Y. Lin, A. Nikolaenko, V. Raghunathan, and E. O. Potma, Adv. Opt. Photon. 3, 1 (2011).

[13] W. P. Beeker, P. Groß, C. J. Lee, C. Cleff, H. L. Offerhaus, C. Fallnich, J. L. Herek, and K.-J. Boller, Opt. Express 17, 22632 (2009).

[14] W. P. Beeker, C. J. Lee, K.-J. Boller, P. Groß, C. Cleff, C. Fallnich, H. L. Offerhaus, and J. L. Herek, Phys. Rev. A 81, 012507 (2010).

[15] B. Schrader and W. Meier, Raman/IR Atlas (Verlag Chemie, Weinheim, 1974).

[16] M. Schmitt, G. Knopp, A. Materny, and W. Kiefer, J. Phys. Chem. A 102, 4059 (1998).

[17] R. F. Dallinger, S. Farquharson, W. H. Woodruff, and M. A. J. Rodgers, J. Am. Chem. Soc. 103, 7433 (1981).

[18] E. J. Heilweil, R. R. Cavanagh, and J. C. Stephenson, Chem. Phys. Lett. 134, 181 (1987). 
[19] T. A. Cellucci and E. R. Nixon, J. Phys. Chem. 89, 1991 (1985).

[20] M. R. Lewis, T. Marshall, E. H. Carnevale, and F. S. Zimnoch, Phys. Rev. 164, 94 (1967).

[21] A. J. Wurzer, T. Wilhelm, J. Piel, and E. Riedle, Chem. Phys. Lett. 299, 296 (1999).

[22] F. Vallée and F. Bogani, Phys. Rev. B 43, 12049 (1991).

[23] T. A. Klar, S. Jakobs, M. Dyba, A. Egner, and S. W. Hell, Proc. Natl. Acad. Sci. USA 97, 8206 (2000).

[24] E. Rittweger, D. Wildanger, and S. W. Hell, Europhys. Lett. 86, 14001 (2009).
[25] E. O. Potma, C. L. Evans, and X. S. Xie, Opt. Lett. 31, 241 (2006).

[26] Y. Fu, H. Wang, R. Shi, and J.-X. Cheng, Opt. Express 14, 3942 (2006).

[27] C. Ventalon, J. M. Fraser, M. H. Vos, A. Alexandrou, J.-L. Martin, and M. Joffre, Proc. Natl. Acad. Sci. USA 101, 13216 (2004).

[28] H. Kamada, H. Gotoh, J. Temmyo, T. Takagahara, and H. Ando, Phys. Rev. Lett. 87, 246401 (2001) 